\title{
Preventing dengue epidemics: how to better assist holistic vaccination efforts
}

\author{
Ibrahim Dadari \\ United Nations Children's Fund, Pacific Office, Honiara Solomon Islands
}

Correspondence: Ibrahim Dadari, United Nations Children’s Fund, Pacific Office, Honiara Solomon Islands, Email ibrahimddr@gmail. com

Received: July 23, 2018 | Published: July 25, 2018

Copyright@ 2018 Dadari et al. This is an open access article distributed under the terms of the Creative Commons Attribution License, which permits unrestricted use, distribution, and reproduction in any medium, provided the original author and source are credited.

\begin{abstract}
The global dengue disease burden is estimated to affect about 400 million people with about a quarter manifesting clinically. Recently, the first vaccine CYDTDV (Dengvaxia) was recommended and licensed for use in individuals $>9$ years of age based on findings from clinical trials; while the highest mortality is seen in children less that 9years of age. The vaccine was also recommended in settings with high sero-prevalence ( $>=70 \%)$ of dengue disease; with recommendations made for a pre-vaccination screening. This paper analyses the determinants and enablers for dengue vaccination needed to control the rising dengue epidemic, if maximizing public health impact remains imperative.
\end{abstract}

Keywords: rash, joint and muscle pains, mild bleeding, dengue shock syndrome, dengue vaccine

\section{Abbreviations:}

DHF, dengue hemorrhagic fever; WHO, world health organization; ICER, incremental cost effectiveness ratio; RDT, rapid diagnostic test

\section{Background}

Dengue virus is a leading cause of morbidity and mortality with an estimated 400million people infected annually worldwide, while only about one-quarter of cases manifesting clinically. ${ }^{1}$ Clinical features are non-specific and include; high grade fever, headache, rash, joint and muscle pains, bone pains, and mild bleeding. The rising trend of the disease could be attributed to globalization, trade, travel, demographic trends, and warming temperatures that result in the recent spread of the primary vectors Aedes aegypti and Aedes albopictus. ${ }^{2}$ Therefore climate change is considered an important determinant of dengue spread. Improved reporting and diagnostic techniques may also play an important role in increasing number of cases being reported. Dengue is currently endemic in over 100 countries with about 3.9billion people at risk. ${ }^{3}$

Dengue- a Flavi virus, has four serotypes. ${ }^{1-4}$ Infection with one of the serotypes does not confer protection from other serotypes; as such people are prone to multiple infections in their lifetime with an increased risk of dengue hemorrhagic fever (DHF) or dengue shock syndrome. ${ }^{1}$ For some not well-understood reasons, second dengue infections tend to be more severe than the first.

Until recently, the mainstay of disease management has been vector control and proper treatment of patients. In July 2016, the World Health Organization (WHO) released the first position paper following successful clinical trials and registration of CYD-TDV vaccines (Dengvaxia) produced by Sanofi Pasteur. ${ }^{4}$

\section{Dengue vaccine(s)}

There are a number of dengue candidate vaccines currently undergoing clinical trials. The WHO vaccine pipeline tracker shows candidate vaccines TDV by Takeda pharmaceutical and TV003/TV005 by the National Institute of Allergy and Infectious Diseases (NIAID) \& Butantan Institute are currently at stage 3 of clinical trials, while CYD-TDV by Sanofi ${ }^{5}$ has been licensed. ${ }^{6}$ All these three vaccines are tetravalent targeting all four serotypes of dengue virus. Other candidate vaccines (such as TDEN, TDENV-PIV and V180) are either in stage 1 or 2 of clinical trials.

CYD-TDV is a live attenuated tetravalent recombinant vaccine administered $0.5 \mathrm{ml}$ as a 3-dose schedule given 6months apart and recommended for individuals 9-45years or 9-60years of age (depending on the license), living in dengue endemic areas. The lower limit of the indication at 9 years of age was chosen due to a safety concern in children aged 2-5years identified in the Phase 3 clinical trials. ${ }^{4}$ CYD-TDV comes as a freeze-dried powder of single or multiple (five) dose vaccine, which needs to be reconstituted and discarded at the end of the session or after 6hours whichever comes first. ${ }^{7}$ The vaccine should be stored at temperatures between $+2{ }^{\circ} \mathrm{C}$ and $+8^{\circ} \mathrm{C}$ like most other vaccines. Dengue vaccine co-administered with other vaccines showed no safety issues. Contraindications are similar to those of live attenuated vaccines. Modeling studies have shown the vaccine to be cost effective at USD2000 per DisabilityAdjusted Life Year at a sero-prevalence of $50-90 \%$ from a societal perspective, if cost is less than USD100-USD150 per fully vaccinated person. ${ }^{4}$ For greater public health impact and cost effectiveness, the WHO recommends vaccination in settings with sero-prevalence of $>=70 \%$ in the target age range 9-45years or 9-60years. WHO does not recommend vaccination with CYD-TDV vaccines in children $<9$ years 
of age due to increased risk of severe dengue and hospitalization in the age group $2-5$ years. $^{4}$

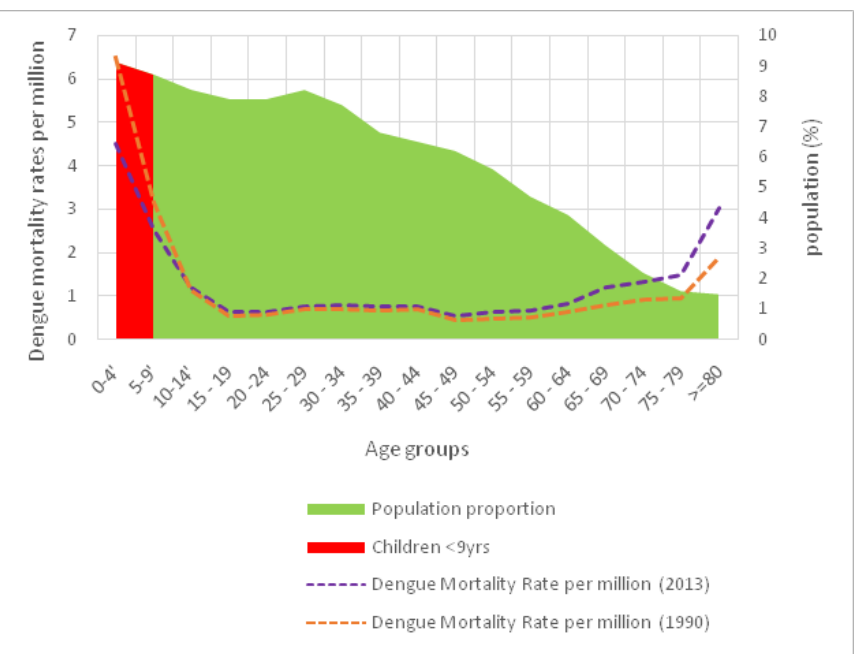

Figure I Showing children under 9yrs of age have highest dengue mortality and highest population as compared to other age groups.'

\section{Practical vaccination issues}

As at April 2016, before the WHO issued the first dengue vaccine position paper, 4countries-Mexico, Brazil, El Salvador and the Philippines-have licensed CYD-TDV vaccines, and moved ahead with vaccine introduction plans. ${ }^{1}$ By December 2017, 19 countries have licensed the vaccine of which 11 have introduced. Based on further analysis of clinical data using a new assay technique developed by the University of Pittsburgh which uses anti-dengue NS1 monoclonal antibodies, looking at long-term efficacy and safety of the vaccine in seronegative and seropositive individuals, Sanofi released a press statement restricting vaccine use to seropositive individuals; while stressing that seronegative individuals have more likelihood of severe infections on the long term upon subsequent infection. ${ }^{5}$ The clinical severity in the vaccinated seronegative group was similar to that of severe cases in the unvaccinated seropositive group. In response to the new data available, WHO provided an updated Questions and Answers related to the dengue vaccine Dengvaxia ${ }^{\circledR}$ and its use in December 2017, and subsequently released a revised Statutory Advisory Group of Experts on Immunization ( $S A G E$ ) recommendations on the vaccine in April 2018. ${ }^{8}$

The revised $S A G E$ recommendations stipulate that for countries considering rolling out CYD-TDV, a pre-vaccination screening strategy should be instituted which will enable only seropositive individuals to be vaccinated. In moderate to high transmission settings, current available rapid diagnostic test (RDT) with low specificity and sensitivity can suffice, where as in low transmission settings a screening test with high specificity is more appropriate. An appropriate screening IgG ELISA test with high sensitivity and specificity can be considered in some settings.

The revised $S A G E$ recommendations, in line with the recent available data, very much fine-tunes requirements for a successful dengue vaccination that is high impact. Most countries with high dengue prevalence however are resource constrained, and therefore will need substantial financial and technical support to institute an efficient screening program pre-vaccination. The additional pre-screening requirement adds to the cost of vaccination per person and could potentially increase the value of the incremental cost effectiveness ratio (ICER) making the vaccine not cost effective in some low and middle-income settings. Additional costs are not only for procuring the screening test kits but also for the logistics of training health workers, distribution, transportation and warehousing of supplies which often is an issue in low resource settings.

Philippines being one of the four countries that earlier went ahead with the introduction of the Dengue vaccine before the release of the WHO position paper in July launched a parliamentary investigation early 2018 into circumstances surrounding the approval, procurement, administration and post marketing surveillance of the dengue vaccine. This may not be unconnected with the press statement released by Sanofi $^{5}$ in November 2017 clarifying recommendations to screen and vaccinate seropositive persons and investigation.

\section{Dengue in children}

Children especially those $<9$ years of age suffer from the highest mortality of Dengue disease. Mortality rates per million cases averages 5.24 in the post neonatal period, 3.75 in children aged 1-4years, 2.53 in age bracket 5-9years and 1.19 in children aged 10-14years. ${ }^{9}$ All other age brackets have mortality rates $<1$ per million. Ironically, the age bracket with the highest mortality cannot receive CYD-TDV vaccines to protect against dengue disease due to safety concerns as observed in clinical trials. Although this is for the safety of children not to receive the vaccines, but it further alienates children from being protected from dengue disease, and therefore calls for more research towards achieving a universally applicable vaccine or most especially a vaccine targeting the most disadvantaged group with the highest dengue mortality (children).

\section{Discussions}

Progress has been made with the registration and use of the first dengue vaccine (CYD-TDV), and the continued clinical trials for other candidate vaccines at advanced stages of development. It is heartbreaking to see that the most vulnerable- children less than 9 years old- are not eligible for the current available vaccine. These age brackets; post neonatal period, 1-4years and 5-9years, have the highest mortality from Dengue vaccines..$^{9}$ Globally the proportion of children $<9$ years of age is estimated at $18 \%,{ }^{10}$ and even higher in high burden dengue countries such as the Philippines where $<9$ years of age constitute about 22 percent of the total population, ${ }^{11}$ and even more in west Africa where the proportion of under 9years is estimated at $31.2 \% .^{10}$ This even becomes more complicated as most children in some of the dengue high burden countries have no birth certificates to verify their ages. It should therefore remain a public health priority that the development and approval of a dengue vaccine that will cover children, most especially the excluded $<9$ years of age, should be fasttracked. This will help reduce the already disproportionate mortality suffered by this age group and reduce the overall dengue mortality.

Introduction of new vaccines remains a challenging task especially in the context of weak health systems. The needed preparations, monitoring and post introduction surveillance and follow up has always required technical assistance to ensure its success, even with the glaring benefits that accompanies the vaccine in the following years. To introduce Dengvaxia, countries are required to check 
sero-prevalence by conducting pre-vaccination screening; ${ }^{8}$ which is crucial in identifying individuals that will benefit from CYD-TDV vaccines, thereby limiting chances for severe secondary dengue. This requirement, will add to the resource (human and material) and technical assistance needs of countries. Designing a package of technical assistance bundled with screening supplies to countries who express their wish to introduce Dengvaxia will go hand in accelerating vaccine introductions. Potentially, introducing dengue surveillance in moderate to high burden countries will ensure availability of seroprevalence data when introduction decisions are made. A stronger post marketing surveillance is needed to maximize benefits of the vaccine to populations. All efforts should continue while employing other vector control measures and proper disease management protocols.

\section{Conclusion and recommendations}

The next dengue position paper will be coming out this September 2018, which will further distill the requirements for vaccination using CYD-TDV, thereby proving updated guidance. It will be very helpful to see a holistic package of technical assistance and screening supplies for countries wishing to introduce the vaccine, and very importantly to see efforts being made towards providing support to candidate vaccines showing promise towards a more holistic dengue vaccination which included children $<9$ years of age.

\section{Authors and contributors}

Ibrahim Dadari, MD, MIHMEP, United Nations Children's Fund, Pacific Office, Honiara Solomon Islands

\section{Acknowledgement}

None.

\section{Disclaimers}

Contents of this article represent my personal observations and have no way connection with my institutional affiliation.

\section{Conflict of interest}

The author declare no conflict of interest.

\section{References}

1. Dengue, United States Centers for Disease Control publication.

2. Kristie L Ebie, Joshua Nealon. Dengue in a Changing Climate. Environmental Research. 2016;151;115-123.

3. Dengue and Severe Dengue. A publication of the World Health Organization; 2018.

4. Dengue vaccine: WHO position paper-July 2016. WHO. 2016;91:349364.

5. Sanofi Updates information on dengue vaccine- press release; 2017.

6. WHO Vaccine pipeline tracker; 2018.

7. WHO Policy Statement: Multi-dose Vial Policy (MDVP). Geneva, Switzerland: World Health Organization; 2014.

8. Revised SAGE recommendation on use of dengue vaccine. 2018.

9. Stanaway JD, Shepard DS, Undurraga EA, et al. The global burden of dengue: an analysis from the Global Burden of Disease Study 2013. Lancet Infect Dis. 2016;16(6):712-723.

10. Population Pyramids of the World 1950 to 2100. 2017.

11. The Age and Sex Structure of the Philippine Population: (Facts from the 2010 Census). Philippines statistics authority. 2012. 